\title{
Sinergia entre extensión y docencia en la Facultad de Ciencias Agropecuarias de Universidad Nacional de Córdoba
}

\author{
Guillermo Ferrer \\ guillermoferre@gmail.com
}

Mario Barrientos

barrientosmarioa@gmail.com
Docentes investigadores de la Facultad de Ciencias Agropecuarias. Universidad Nacional de Córdoba, Argentina
Integración de la docencia y la extensión /

Intervenciones

RECEPCIÓN: 24/06/16

ACEPTACIÓN FINAL: 10/10/16

\section{Resumen}

Este trabajo aborda una experiencia realizada por un grupo de universitarios que, en parte, comparten dos proyectos, uno docente vinculado a la constitución de un "área de consolidación" con la cual los estudiantes de agronomía finalizan sus estudios, y otro de extensión a partir de un proyecto de intervención que, desde una de sus líneas de trabajo, brinda apoyo a unidades de producción familiar en transición agroecológica, organizadas en ferias francas.

Podemos concluir que se ha generado una importante sinergia entre el proyecto de extensión y el espacio curricular de la citada área de consolidación. Por el lado del proyecto de extensión, se ve fortalecido porque los productores reciben propuestas de posibles modificaciones tendientes a mejorar su emprendimiento y desde el aspecto pedagógico se cuenta con unidades de producción que posibilitan a los estudiantes la elaboración de una propuesta técnico-productiva que constituye una verdadera síntesis de carrera.

Palabras-clave

- Docencia universitaria

- Extensión universitaria

- Agroecología

- Productores familiares

- Córdoba

\section{Resumo}

Este artigo trata de uma experiência feita por um grupo de estudantes universitários que, em parte, compartilham dois projetos, um deles docente, ligado à criação de uma "área de consolidação" com que os estudantes de agronomia concluem seus estudos, e outro de extensão, a partir de um projeto de intervenção que, de uma de suas linhas de trabalho, apoiam unidades de produção familiar em transição agroecológica, organizadas em feiras livres. Podemos concluir que se gerou uma sinergia importante entre o projeto de extensão e o espaço curricular da citada área de consolidação. O projeto de extensão se vê fortalecido porque os produtores recebem propostas de possíveis mudanças destinadas a melhorar o seu empreendimento, e do ponto de vista pedagógico, conta com unidades de produção que fazem possíveis os alunos desenvolverem uma proposta técnico-produtiva que constitui uma verdadeira síntese do curso.

Palavras-chave

- Docência universitária

- Extensão Universitária

- Agroecologia

- Produtores Familiares

- Córdoba

\section{Para citación de este artículo}

Ferrer, G. y Barrientos, M. (2016). Sinergia entre extensión y docencia en la Facultad de Ciencias Agropecuarias de Universidad Nacional de Córdoba (Argentina). En Revista $+E$, (6), pp. 248-255. Santa Fe, Argentina: Ediciones UNL. 


\section{Introducción}

Este trabajo aborda una experiencia realizada por un grupo de universitarios que, en parte, comparten dos proyectos, uno docente vinculado a la constitución de un "área de consolidación" con la cual los estudiantes de agronomía finalizan sus estudios y otro de extensión, a partir de un proyecto de intervención que, desde una de sus líneas de trabajo, brinda apoyo a unidades de producción familiar en transición agroecológica organizadas en ferias francas. Por el lado de la extensión, acordamos con los planteos de Peralta (2010), quien entiende la extensión universitaria como espacio de encuentro entre actores universitarios y otros miembros de la comunidad que, a través de la comunicación y el intercambio de conocimientos, promueve un proceso educativo, cultural y científico articulándose con la docencia y la investigación, lo cual posibilita una relación transformadora de la realidad.

Dado que transformar la realidad requiere un esfuerzo mancomunado de todos los sujetos intervinientes, buscamos desarrollar, como agentes universitarios, nuestra capacidad de diálogo con actores de diversos grupos culturales y variadas realidades y aumentar nuestra capacidad de comprensión para proponer intercambios de conocimientos adecuados a las posibilidades y expectativas de los actores del territorio y pertinentes a los problemas que se decida enfrentar. Esta búsqueda metodológica se enmarca en la extensión agroecológica (Alemany y Sevilla Guzmán, 2007). La extensión rural convencional fue generada para impulsar la "modernización agraria", sostenida conceptualmente por el trabajo conductista de Rogers y Shoemaker (1971). Esta propuesta acompañó la revolución verde y su proceso de concentración y exclusión de muchos productores, que llevó al lamentable "desierto verde" que tenemos principalmente en las zonas agrícolas, ya que quedan pocas familias viviendo en el campo.

La otra extensión, que podríamos denominar alternativa, se conecta con las propuestas de Chayanov de comienzos del siglo XX (Shanin, 2006) sobre la evolución de la agricultura pero respetando la participación del campesinado. Otro hito central es el de la educación popular de Freire (1969) que busca la concientización de los productores, para generar personas más críticas y con mayores capacidades de actuar socialmente. Conectado con la educación popular surge, también desde Latinoamérica, la Investigación Acción Participativa (Fals Borda y Rodrigues Brandao, 1986) como una forma de profundizar el conocimiento junto a la gente, ya que las preguntas de esa investigación surgen y fundamentan la acción de transformación de la realidad. En estos postulados es que se inscribe la actual perspectiva teórica de la extensión agroecológica que se enriquece con marcos teóricos aportados por la ecología política y la economía ecológica (Leff 1986; Naredo 2006) para hacer una lectura integral del sistema mundo, y desde lo metodológico, define su práctica como investigación acción participativa, en un diálogo de distintos saberes (científico-popular) que puede ser conceptualizado en la dimensión educativa desde la pedagogía constructivista.

En otras palabras, la extensión agroecológica aborda integralmente los problemas socioproductivos reconociendo la diversidad de actores presentes en el territorio y reconociendo sus saberes con sus distintas epistemologías y formas de conocimiento, con lo cual se amplían las capacidades de interpretación y resolución de los problemas complejos que plantea el territorio.

Abordar la realidad en forma integral, como propone la extensión agroecológica, demanda un trabajo interdisciplinario y la puesta en tensión de conceptos y modelos que deberán demostrar su pertinencia para explicar los problemas de la realidad. El desafío es enfrentar los conceptos promovidos por las grandes transnacionales de la alimentación y de la agricultura industrial, proponiendo un modelo de transición hacia una agricultura agroecológica que es la que nos provee de alimentos sanos y generados en relaciones justas, tanto de producción como de comercialización.

Esta forma de ver la producción agrícola, se puede encontrar incluida en el concepto de soberanía alimentaria introducido por la organización Vía Campesina (2003, s/n), que plantea que la "soberanía alimentaria es la facultad de cada pueblo para definir sus propias políticas agrarias y alimentarias, de acuerdo a 
objetivos de desarrollo sostenible". Ello implica la protección del mercado doméstico contra los productos que se venden a precios subsidiados en el mercado internacional.

En coincidencia con el concepto planteado por Vía Campesina, Caeiro (2013), propone la inclusión de un enfoque territorial del desarrollo que favorece el reconocimiento de una concepción de continuo espacial, de integración de mercados, de redes sociales, de instituciones y de cultura que integran lo urbano con lo rural. El conocimiento, la actitud y la responsabilidad ante la propia alimentación son actos políticos, de soberanía alimentaria. Educar-nos para alimentar-nos con dignidad y de forma saludable, teniendo en cuenta las consecuencias de nuestra elección, es una tarea necesaria, hoy más que nunca.

Lo anteriormente expresado exige una inserción comunitariaterritorial por parte del equipo extensionista que, en conjunto con los actores extra-universitarios constituyen el soporte de este proyecto, para alcanzar uno de los objetivos meta que es la generación de procesos de participación colectiva, que permitan la re-creación y re-significación de una identidad comunitaria del grupo de productores y sus familias y la visibilidad externa de su capital social como comunidad organizada.

Por el lado docente, entendemos al aprendizaje como un cambio, modificación o transformación del comportamiento de un sujeto, de relativa permanencia, que ocurre como resultado de la práctica. De esta forma, promover el aprendizaje implica organizar una serie de acciones orientadas hacia la meta propuesta, ya que una persona aprende cuando se plantea dudas, formula hipótesis, retrocede ante ciertos obstáculos, arriba a conclusiones parciales, siente temores ante lo desconocido, intolerancia frente a contenidos y experiencias nuevas, verifica en una práctica sus conclusiones, etc., acciones todas que definen la idea de proceso $y$, por lo tanto, implican que iniciar un proceso de conocimientos es ponerse en movimiento (Barrientos y Ryan, 2015).

Desde esta concepción constructivista se hace necesario que los estudiantes puedan interactuar directamente con el objeto de estudio, ya que de su problematización y participación depende la posibilidad de establecer aprendizajes significativos. Ellos deben ser desafiados por la complejidad de la realidad y movilizados por el de modificarla con propuestas fundamentadas para mejorar los sistemas productivos en el camino de la sustentabilidad.

De acuerdo con Ávila (2015), decimos que cuando hablamos de educación nos estamos refiriendo a situaciones donde se ponen en juego los saberes y conocimientos que permiten relacionarse de nuevos modos con el mundo natural y social. Los procesos educativos se configuran en las redes de los intercambios sociales siempre que están en juego los procesos del conocer en el seno de una trama discursiva construida con otros con el sentido de "mostrar el mundo" con sus diferentes acervos culturales buscando lograr un cierto grado de reflexión respecto de la misma.
Coincidimos con las ideas que Pablo Freire expuso en su célebre texto Extensión o comunicación, donde sienta las bases de sus perspectivas acerca de la educación como situación gnoseológica atravesada por la problematización acerca del mundo social en el seno de una construcción dialógica. Co-construcción que supone un trabajo de desnaturalización de las miradas tanto de los campesinos como de los agrónomos planteando la necesidad de la crítica y la reflexión acerca del conocimiento del mundo y del lugar de estos actores en esos procesos de conocimiento y transformación. El planteo de Freire apunta a pensar la educación como una experiencia gnoseológica configurada dialógicamente y con un sentido profundamente político y crítico: intervención con otros en el mundo, convicción de que el cambio es posible y de que es necesario para ello el conocimiento crítico de la realidad y la elucidación de las condiciones sociales que la estructuran. Pero es importante destacar que el aporte educativo de la perspectiva freireana no se agota en su posicionamiento en el cambio, sino que realiza interesantes reflexiones acerca de las relaciones pedagógicas, del papel del conocimiento y su construcción, del lugar del educador y del educando, de los problemas educativos emergentes tanto en las prácticas sociales educativas, como en las instituciones, en las que considera imprescindible reconocer los múltiples espacios de educación que ofrecen más allá del aula y el papel central de los docentes, en tanto puedan asumirse en este lugar activo y de intervención y revisar críticamente su práctica. En este contexto, entendemos la intervención educativa como la construcción de propuestas que a partir del análisis de los procesos sociales y culturales en los que se inserta y la clara identificación de los sujetos, organice la puesta en juego de saberes de distinta naturaleza y especificidad, con el objetivo de generar procesos de circulación, transmisión, apropiación y resignificación de esos saberes, y elabore las estrategias metodológicas y prácticas que den cauce a los procesos educativos esperados, acompañando y redefiniendo los cursos de acción en las instancias en que sea necesario (Ávila, 2015). La educación en espacios extra áulicos involucra aquellas intervenciones gestadas y situadas en la trama de procesos sociales y culturales de inscripción local, contenidos en redes de relaciones delimitadas territorialmente, lo cual supone un espacio de interacciones cotidianas y relaciones cara a cara producidas en el cruce de trayectorias entrelazadas y encuentros subjetivos y sociales acuñados en la urdimbre de esos procesos, sus conflictos y tensiones. En esa perspectiva, resulta necesario, por consiguiente, abordar los espacios y movimientos productivos, sus lógicas y complejidades, a fin de situar y comprender las tramas en las que las prácticas educativas se conforman y despliegan, así como problematizar sus sentidos, orientaciones y estrategias. En palabras de Ávila (2015), el agente de la educación, aquel sujeto que asume la tarea educativa, cumple la función de proponer, 


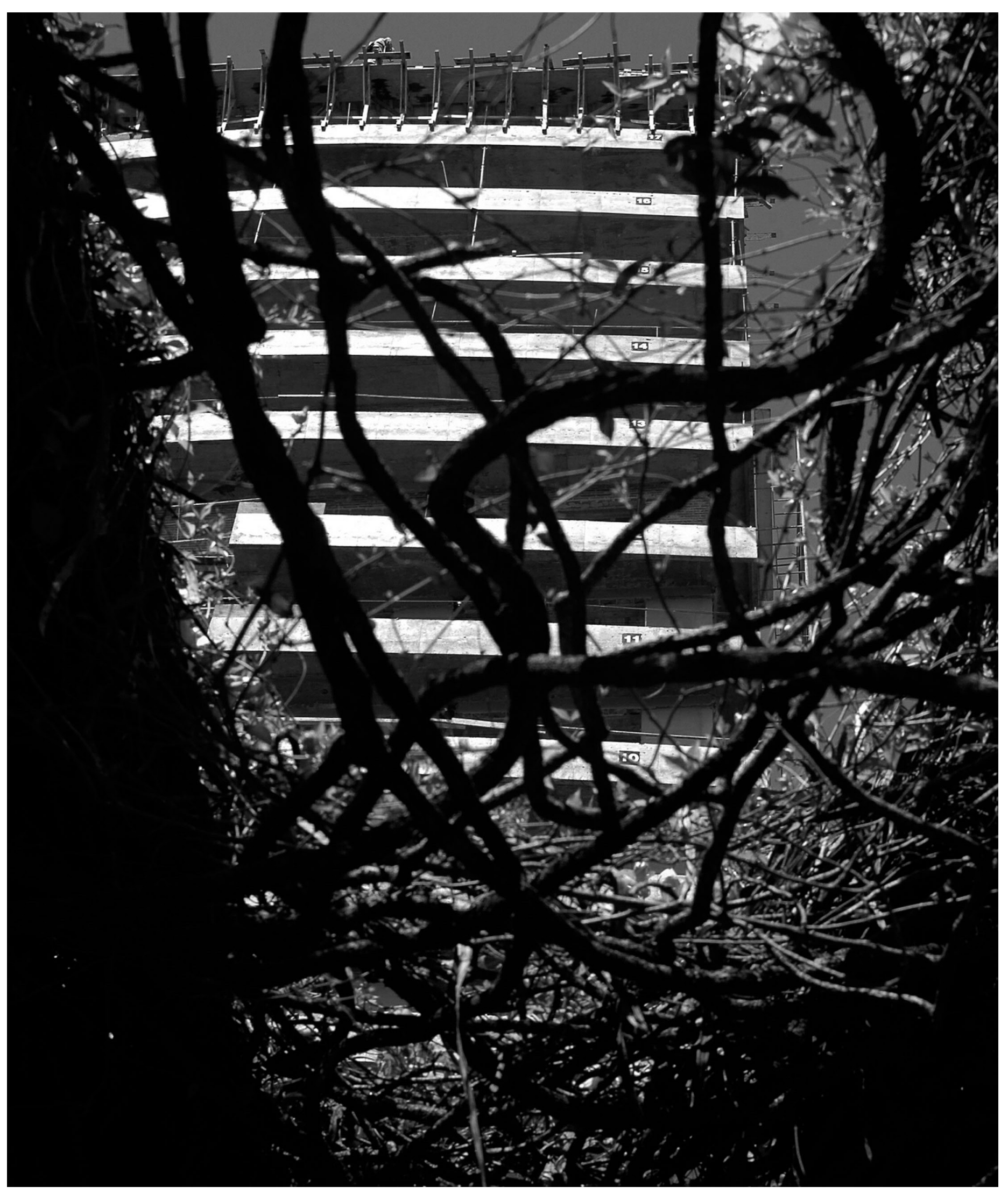

(c) Hugo Pascucci 
construir condiciones, anticipar procesos, organizar estrategias, para abrir nuevas puertas a la cultura de la época, nuevos espacios de vinculación y participación, nuevas filiaciones a través del acceso a contenidos culturales y recursos para interpretar el mundo en sus diversas complejidades. En este sentido, enfatizamos la necesidad de un trabajo de construcción crítica que implica el reconocimiento de los sujetos, posicionamientos en su práctica y saberes específicos que le permitan organizar esos procesos y orientarlos. En palabras de Paulo Freire, se trata de pensar, reflexionar y elaborar "situaciones creadoras de saberes", teniendo en cuenta los elementos claves de la situación educativa.

\section{La extensión universitaria}

El proyecto de extensión es ejecutado por docentes, estudiantes y egresados de la Facultad de Ciencias Agropecuarias y la Escuela de Nutrición, por personal de la Agencia de Extensión Córdoba del INTA y de la Secretaría de Agricultura Familiar. Su implementación pretende contribuir a fortalecer la soberanía alimentaria, como derecho de las personas, apoyando procesos de producción agroecológica en unidades de producción familiar urbanas y periurbanas dedicadas a la producción de hortalizas mediante la generación de espacios de comercialización justa y la promoción del consumo de alimentos sanos.

Los principales objetivos son:

- Capacitar a los productores huerteros en producción agroecológica y estimular la organización de la producción a una escala mayor. - Acompañar el proceso de organización social de los huerteros para el afianzamiento de su participación en ferias.

- Revalorizar y generar, con los huerteros y sus familias, estrategias de producción agroecológica, criterios de alimentación saludable y un concepto de salud desde su identidad cultural y significados socioculturales como medio para mejorar la soberanía alimentaria del sector.

Estos objetivos se vinculan con algunos de los aspectos señalados por de Souza Santos (2005), quien afirma que es necesario marcar distancia con orientaciones hegemónicas de los últimos 20 años y concebir las relaciones "de modo alternativo al capitalismo global, atribuyendo a las universidades una participación activa en la cohesión social, en la profundización de la democracia, en la lucha contra la exclusión social, la degradación ambiental y en la defensa de la diversidad cultural".

Para abordar este proyecto se desplegó una estrategia que involucró distintos actores del territorio, por lo que se organizaron cuatro grupos de trabajo con objetivos específicos, pero aportando centrípetamente en la concreción del objetivo general, a saber: i) Feria Agroecológica de Córdoba, ii) escuelas primarias, iii) comunicación masiva vía radios locales, y iv) huertas serranas agroecológicas.
La problemática de la soberanía alimentaria que se contempla en este proyecto se centra en dos dimensiones principales: la oferta alimentaria y la demanda. En cuanto a la primera, los huerteros tienen dificultades para ampliar su escala, pasando de una huerta familiar a una con excedentes comercializables y para organizarse para participar de ferias. En lo que concierne a "los consumidores", los ciudadanos presentan un grado importante de confusión al momento de tener que decidir o diferenciar entre los alimentos diet o light — supuestamente saludables — que ofrece la industria de la alimentación y alimentos o productos sanos y naturales.

La Feria Agroecológica de Córdoba (FAC), en cambio, ofrece alimentos producidos de manera agroecológica por los propios feriantes, como hortalizas, frutas, huevos, miel, aceite de oliva, especias, brotes, plantines de especies hortícolas y aromáticas y de árboles autóctonos elaborados por ellos mismos, a partir de materias primas agroecológicas, como pastas secas, panificados, mermeladas, harinas y productos cosméticos. También se ofrecen compost y fertilizantes y alimentos que no se producen localmente pero que cuentan con certificación agroecológica u orgánica, como granos, semillas, harinas, té y yerba. Los feriantes son productores familiares que, en las limitadas superficies que poseen, instalan huertos biodiversos que atienden aportando su propia fuerza de trabajo, y que cuentan con el acompañamiento y asesoramiento del Programa Pro Huerta del Instituto Nacional de Tecnología Agropecuaria (INTA) y/o la Secretaría de Agricultura Familiar, instituciones que conjuntamente con la Universidad Nacional de Córdoba y organizaciones de productores, conforman la Mesa de Agricultura Urbana de Córdoba, espacio desde el cual se gestó la FAC y que promueve la producción agroecológica de alimentos. Los consumidores están cada vez más concientizados acerca de la preservación de la salud, y poseen un mayor conocimiento sobre los problemas sanitarios que provocan los alimentos tratados con agrotóxicos o con formulaciones de síntesis química no saludables (conservantes, por ejemplo). La industria también ha observado esta tendencia alimentaria y ofrece con un fuerte marketing de alimentos saludables una gran cantidad de productos supuestamente benéficos, pero oculta las sustancias dañinas o potencialmente dañinas que contienen.

El ámbito de la FAC se constituye así en un espacio propicio para la intervención con miras a la formación de los ciudadanos en aspectos agroecológicos dada, por una parte, su riqueza social, productiva y cultural al albergar una amplia diversidad tanto de productores y producciones familiares como de consumidores, y por la otra por constituirse en una "vidriera" para quienes tienen intenciones de iniciarse en la producción agroecológica y/o aumentar su escala de producción con vistas a generar excedentes comercializables de verduras y hortalizas.

La producción agroecológica que es comercializada en la FAC tiene lugar en sistemas productivos del periurbano de la ciudad de Córdoba que son espacios de intervención tanto desde el proyecto 
desafío del equipo extensionista es

compatibilizar la soberanía alimentaria, la salud ambiental y humana, la

conservación de los recursos naturales, y avanzar en la inclusión social de extensión como de las actividades docentes planteadas desde el Área de Consolidación Agroecología y Desarrollo Territorial. Por ello, el desafío del equipo extensionista es compatibilizar la soberanía alimentaria, la salud ambiental y humana, la conservación de los recursos naturales, y avanzar en la inclusión social de las familias y de las comunidades involucradas en el proyecto.

\section{La docencia universitaria}

En cuanto a la enseñanza universitaria, el Plan de Estudio 2004 de la FCA incluye un nuevo espacio curricular denominado "áreas de consolidación". En el último semestre de su carrera, los estudiantes optan por un área de conocimientos en la que refuerzan sus aprendizajes. Existen ocho áreas posibles de ser elegidas y una de ellas es el Área de Agroecología y Desarrollo Territorial. La misma estructura sus módulos de contenidos en tres bloques. El primero, dedicado al sistema productivo, el segundo al territorio y el final a las políticas nacionales que atienden la problemática de los productores familiares.

Desde el primer módulo se asigna a un grupo de hasta tres estudiantes un sistema productivo en transición agroecológica. Este sistema se convierte en la unidad de análisis, en la base empírica para que los estudiantes vayan estableciendo relaciones entre los datos de la realidad y la teoría a lo largo del cursado, tanto en los aspectos productivos, como en los comerciales y organizacionales que aparecen en el territorio y que terminarán en la elaboración de un Trabajo Académico Integrador (TAl) con el cual concluyen la carrera, a cuya presentación asisten las familias productoras. La relación es clara: las unidades de producción agroecológica que los estudiantes abordan y sobre las cuales realizan su TAI son las de los huerteros/feriantes que son acompañados desde el proyecto de extensión universitaria. En la cohorte 2015, 25 estudiantes optaron por el Área de Agroecología y Desarrollo, los que conformaron diez grupos que abordaron una unidad de producción cada uno. Los sistemas productivos correspondían a dos de granja, uno frutícola, uno de producción de trigo, uno de ganadería caprina y cinco hortícolas.

De esta forma los procesos de extensión universitaria se ven apoyados por los estudiantes a la vez que abren nuevos espacios educativos para la docencia. Así se posibilita una relación sinérgica entre la docencia y la extensión, ya que exponer, fundamentar y debatir ideas (los conocimientos académicos que le dan sentido a actividad educativa) nos obliga a exponer, fundamentar y debatir prácticas (las acciones de extensión), dado que sostenemos que no existen unas sin las otras.

\section{Conclusiones}

Podemos concluir que se ha generado una importante sinergia entre el proyecto de extensión y el espacio curricular de la citada área de consolidación.

Respecto del proyecto de extensión, se ve fortalecido porque los productores disponen de un grupo de estudiantes con quien repensar sus procesos productivos y comerciales y reciben propuestas de posibles modificaciones tendientes a mejorar su emprendimiento.

Desde el aspecto pedagógico, se cuenta con unidades de producción que los docentes conocen y que posibilitan a los estudiantes la construcción del conocimiento sobre problemas reales que logran movilizarlos, comprometiéndolos a realizar una búsqueda conceptual y la elaboración de una propuesta técnicoproductiva que constituye una verdadera síntesis de la carrera. Además, el hecho de que los productores participen en las presentaciones de los estudiantes, observando sus producciones y propuestas, es un reaseguro de que el trabajo no sólo es auténtico sino también útil.

Otro punto destacable en esta propuesta es su visión de que la integración de la Universidad con la comunidad no se realiza solamente saliendo de los claustros, sino también posibilitando que los productores ingresen a ella. Esto tiene lugar cuando 


\section{6}

\author{
el hecho de que los productores participen \\ en las presentaciones de los estudiantes, \\ observando sus producciones y propuestas, \\ es un reaseguro de que el trabajo no sólo es \\ auténtico sino también útil
}

el grupo de estudiantes expone o defiende su TAl y la familia productora es invitada especialmente a participar en una instancia académicamente destacada.

La Universidad en el territorio, a través de la extensión universitaria, abre posibilidades de aprendizaje al expandir las aulas integrándolas con la problemática real de la comunidad.

La multidimensionalidad del diálogo que promueve esta articulación lleva implícita, y a la vez promueve, la generación de espacios interdisciplinares que al posibilitar la interacción de enfoques, conceptos, modelos y metodologías permiten definir acciones de verdadero impacto social al potenciar la generación de estrategias de promoción del desarrollo individual y social y la definición de políticas públicas, atendiendo a los intereses que las instituciones y organizaciones de la sociedad priorizan.

Aunque generalmente pensamos en la educación formal de los estudiantes y la no formal de los productores, constatamos que la sinergia del proceso también alcanza a los docentes. Desde la docencia asumimos determinado marco conceptual que podemos exponer a los estudiantes y vincular con ejemplos y otras ideas, pero cuando tenemos que aplicarlos en la realidad vemos la complejidad y dificultad que encierra la operacionalización de los postulados teóricos. Tal es el caso de reconocer en el encuentro generado por la extensión las diversas epistemologías de los distintos actores y sus saberes, con lo cual uno acuerda intelectualmente. Pero puesto en situación, por ejemplo, se hace difícil articular propuestas de agricultura biodinámica que sostienen la influencia de los planetas en los cultivos con la formación académica de sesgo positivista. O como es el caso de consensuar las distintas posiciones e intereses de los actores en el marco de las ferias, donde el interés de los agricultores es obtener el mayor valor por sus productos entrando en contradicción con el interés de los consumidores y por lo tanto, la propuesta de un comercio justo postulado desde el concepto de soberanía alimentaria, que demanda asumir una perspectiva de bienestar comunitario general por encima de los intereses individuales. Estas vivencia en el territorio de extensión, interpelan al docente, lo transforman y lo hacen más consiente sobre la implicancia de los conceptos que plantea.

Finalmente, podríamos afirmar que el hecho de que la Universidad se ponga en diálogo con la sociedad implica no solo que sus conocimientos pueden ser mejor aprovechados por la comunidad sino, en el sentido inverso, que tanto los conocimientos que nutren la formación de los profesionales como los que genera son aquellos que la sociedad está necesitando para superar las limitaciones que le impiden desarrollarse. Por ende, se hace casi imprescindible que la extensión se integre no solamente con la docencia sino también con la tercera de las funciones básicas de la universidad, la investigación. 


\section{Referencias bibliográficas}

Alemany, C. y Sevilla Guzmán, E. (2007). ¿Vuelve la extensión rural? Reflexiones y propuestas agroecológicas vinculadas con el retorno y fortalecimiento de la extensión rural en América Latina. Realidad Económica, (227), 52-74.

Ávila, O. (2015). Extensión y educación popular. Contextos, Prácticas y Reflexiones. Compendio Bibliográfico. Asignatura Extensión Universitaria. SEU-UNC.

Barrientos, M. y Ryan, S. (2015). Relación entre extensión educación y comunicación. Compendio Bibliográfico. Asignatura Extensión Rural. FCA-UNC.

Caeiro, R. (2013). Elementos importantes del enfoque territorial del Desarrollo Rural. Recuperado en mayo de 2016 de: http://inta.gob.ar/documentos/elementos-importantes-del-enfoque-territorial-del-desarrollo-rural

Fals Borda, O. y Rodrigues Brandao, C. (1986). Investigación participativa. Montevideo: Instituto del Hombre.

Freire, P. (1969). Extensión o comunicación. Santiago de Chile: Instituto de Capacitación e Investigación en Reforma Agraria.

Leff, E. (1986). Ecología y Capital. Racionalidad ambiental, democracia participativa y desarrollo sustentable. Madrid: Siglo XXI Editores.

Naredo, J.M. (2006). Raíces económicas del deterioro ecológico y social. Madrid: Siglo XXI Editores.

Peralta, M. (2010). La Universidad en la construcción de Políticas Públicas. IV Congreso Nacional de Extensión Universitaria. Mendoza. Disponible en: http:// www.uncuyo.edu.ar/extension/upload/Mar\%C3\%ADa_In\%C3\%A9s_Peralta.pdf Rogers, E.; Shoemaker, F. (1971). Communication of innovations: a cross-cultural approach. New York: Free Press.

Souza Santos, B. de (2005). La Universidad en el siglo XXI. Para una reforma democrática y emancipadora de la Universidad. Laboratorio de Políticas Públicas. Buenos Aires: Miño y Dávila.

Shanin, T. (2006). Naturaleza y lógica de la economía campesina. Barcelona: Anagrama.

Vía Campesina (2003). ¿Qué es la soberanía alimentaria? Disponible en: http:// viacampesina.org/es/index.php/temas-principales-mainmenu-27/soberanalimentary-comercio-mainmenu-38/314-que-es-la-soberania-alimentaria (Recuperado 5 de mayo de 2016)

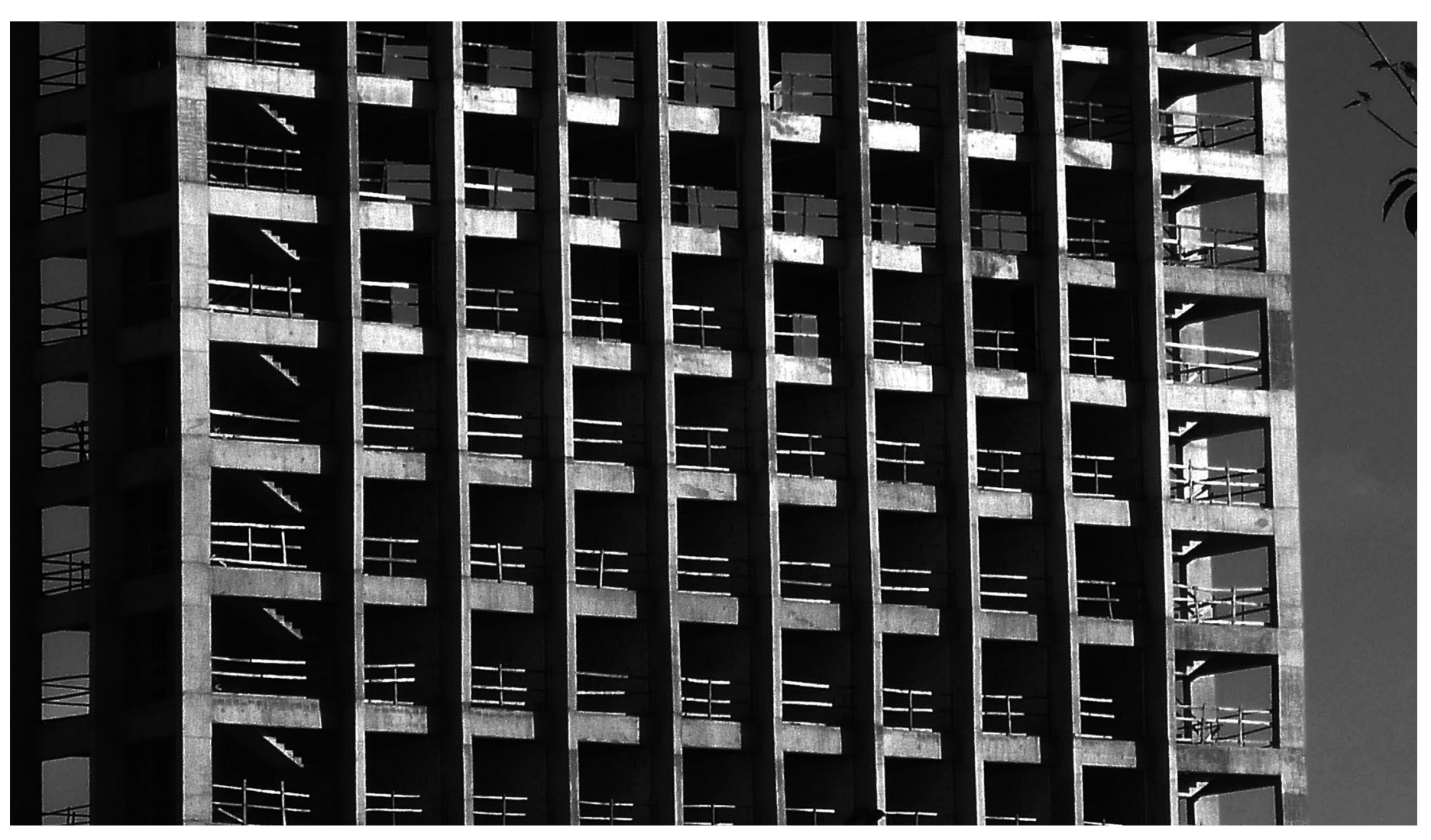

(c) Oscar Dechiara 